\title{
First Technical Specifications for Health Risk Assessment of Ambient Air Pollution in China
}

\author{
Jingxiu Han ${ }^{1}$; Junrui Chang'; Jingyi Liu' ${ }^{1}$; Congshen Meng'; Dongqun $\mathrm{Xu}^{1, \text { t, }}$
}

\begin{abstract}
The Global Burden of Disease study showed that air pollution is the fourth risk factor for attributable deaths (1) and a major public health problem in China. Therefore, the Chinese government has taken a series of measures to control air pollution. However, the attributable disease burden of air pollution can be effectively reduced only by integrating health into air pollution control policies. Health risk assessment comprehensively considered the impact of atmospheric pollution levels, pollutant toxicity, exposure-response coefficients, and the characteristics of exposed population. It may provide a scientific basis for formulating policies for air pollution prevention and control, revising standards of ambient air quality, and putting forward targeted measures for public health protection.
\end{abstract}

\section{BACKGROUND}

A network of air pollution and health impact monitoring was established in 2013 to cope with the impact of severe haze on the health of China's population (2). By 2019, the monitoring network had covered 164 counties in 84 cities in 31 provincial-level administrative divisions on the mainland of China to gather monitoring data on air quality and population health. This, therefore, provided a data basis for health risk assessment.

A comprehensive review of the published literature on health risk assessment found that the four-step evaluation procedure, which includes hazard identification, exposure-response assessment, exposure assessment, and risk characteristic analysis, are the basic framework for carrying out health risk assessments. There were some problems in the process of risk assessment, including poor data quality, blind use of models for assessment, opaque assessment process, lack of uncertainty analysis of assessment results, and incomplete information in risk assessment reports. Health risk assessment techniques need to be standardized to guide relevant staff to conduct the assessment in a scientific, transparent, and consistent manner and to identify the main health hazards and potential health risks of urban air pollution.

Before 2019, the Ministry of Environmental Protection (Ministry of Ecology and Environment of the People's Republic of China) and the Ministry of Health (National Health Commission of the People's Republic of China) issued some technical guidelines for environmental risk assessment to protect ambient air quality, but there were no guidelines for health risk assessment to assess the health risk of air pollution (3). Therefore, there was an urgent need to develop technical specifications for health risk assessment of ambient air pollution in order to guide the local scientific use of monitoring data, to evaluate the current health effects and risks of atmospheric pollution in China, and to provide a scientific basis for effective intervention measures to reduce the health hazards caused by air pollution.

\section{METHODS}

First, the National Institute of Environmental Health of China CDC widely collected international technical documents and publications related to environmental health impact assessments and health risk assessments, including guides, specifications, assessment reports, and evaluation methods and tools. All of these were analyzed comprehensively to understand the characteristics and application scope of various health risk assessment methods and tools. It was determined that the four-step evaluation procedures were also followed in this specification, which was recognized by developed countries and international organizations. The four-steps included hazard identification, exposure-response assessment, exposure assessment, and risk characteristic analysis.

Second, the characteristics of the air pollutants and health data were analyzed by using the monitoring system data, which included not only data on the concentrations of air pollutants related to exposure but 
also data on causes of death, outpatient and emergency hospital visits, hospitalizations related to health, and meteorological data affecting air pollution and health outcomes. Aimed towards the problems emerging in previous risk assessments and drawing lessons from the standards and guides provided in recent years, the "Technical Specifications for Health Risk Assessment of Ambient Air Pollution" (WS/T 666-2019) was formulated to be people-oriented, scientific, feasible, and practical (4). The specifications included three parts: General Provisions, Health Risk Assessments of Populations Based on Demographic Data, and Health Risk Assessments of Populations Based on Toxicity Data of Air Pollutants. Sichuan CDC and Jiangsu CDC jointly participated in the development of the specification.

\section{RATIONALE AND EVIDENCE}

To fully use international research results, the guidelines for health impact assessments and health risk assessments issued by the World Health Organization (WHO) and the US Environmental Protection Agency (US EPA) were adjusted to fit the actual circumstances in China. The technical specifications (WS/T 666-2019) detail and expand relevant content and put forward data quality requirements based on the existing monitoring data in China. Two methods of health risk assessment were given to expand the scope of applications of risk assessments.

One method was based on demographic data, which mainly referenced relevant guidelines, methods, and tools developed by WHO (5-6). As for the assessment of the exposure-response relationship, two methods were given to obtain the coefficient. One was developed using existing literature, and the other was through the analysis of survey or monitoring data. This specification also provided methods for calculating the coefficient of the exposure-response curve. The coefficient obtained by either method for acute or chronic health effects should be selected accordingly, depending on the needs of the health risk assessment.

The other method was based on the concentration of contaminants and their toxicity, which mainly referenced the method recommended by the US EPA (7-11). Based on practical application considerations, the steps of assessing the exposure-response relationship in health risk assessments were defined as follows: obtaining the concentration data of air pollutants, obtaining the exposure parameters of population, consulting the toxicological data of pollutants, and determining the screening concentrations. Screening concentrations were threshold concentrations of health effects of carcinogenic and non-carcinogenic air pollutants selected under specific exposure conditions. If the contaminant concentration was less than the screening concentration, the health risk assessment process could be terminated; otherwise, the health risk assessment needed to continue. In addition, since the toxicity of some pollutants was age-sensitive, this specification introduced age-sensitive factors to calculate the excess carcinogenic risk of air pollutants accurately. The calculation methods of exposure concentration of carcinogenic and non-carcinogenic air pollutants were listed in detail in the exposure assessment. Excess carcinogenic risk assessment, non-carcinogenic risk assessment, and uncertainty analysis were listed in the health risk assessment.

\section{RECOMMENDATIONS}

The technical specifications (WS/T 666-2019) came into effect on January 1, 2020 and were directly applicable to assessing the health risk of air pollutants inhaled into the human body at the local, regional, and national levels. These technical specifications have provided general rules for assessing the health risk of air pollution, including the basic principles of the assessment, the assessment process, the formulation of plans, selection methods, feedback procedures and next steps, implementation of the assessments, sources of uncertainty in the assessments, risk assessment reports, and application of results, etc. The workflow, assessment methods, and requirements of two kinds of assessment methods based on population characteristic data and pollutant toxicity were specified. Appendices were provided, including a case of air pollution population health risk assessment, a report template, etc. These technical specifications can guide evaluators to choose appropriate assessment methods, tools, and data resources that meet the requirements, carry out the health risk assessment, and reasonably interpret the assessment results.

\section{Population-based Health Risk Assessments}

Population-based health risk assessments provided assessment methods by using populationepidemiological data. Acute health risk assessment 
methods can be used to assess the health risks of heavy pollution and phased or temporary air pollution prevention and control. The exposure-response coefficient can be the results of time series and casecrossover studies. The results of chronic health risk assessments are preferred in the formulation of standard limits of air pollutants and long-term targets for the prevention and control of air pollution. The coefficient can be obtained from cohort studies or cross-sectional studies. In addition, coefficients should give preference to results of local or regional similarities in climate, geography, exposure levels, economy, and customs. Exposure assessments should consider the range of concentration of air pollutants people are exposed to and focus on those at high health risks. In risk characterization analysis, the selection of reference concentration should consider not only the purpose of risk assessment, but also the level of economic development and technical feasibility. This method takes into account the exposure of the population and the impact of pollutants on human health and is closer to the real risk of air pollution on human health. However, it is difficult to obtain and accurately evaluate the population data.

\section{Toxicity-based Health Risk Assessments}

Toxicity-based carcinogenic and non-carcinogenic health risk assessments can be conducted when contaminant concentration and toxicity data are available. Age-sensitive factors were introduced into carcinogenic risk assessments, and $1 \times 10^{-6}$ was recommended as the acceptable carcinogenic risk. For non-carcinogenic risks, acute, sub-chronic, and chronic exposures were considered. Hazard quotient one was considered as the acceptable risk of non-carcinogenic air pollutants. In actual scenarios, people are often exposed to multiple air pollutants at the same time, or to one or more air pollutants intermittently. Therefore, cumulative exposure needs to be calculated in exposure assessments. The required parameters in this method are easy to obtain, but since the results based on toxicological data involve extrapolation of experimental results, the uncertainty of population health risk assessment results is greater.

\section{COMMENT}

These technical specifications (WS/T 666-2019) are the first on environmental health risk assessment issued by China's health authorities, which promotes the establishment of a health risk assessment system and facilitates the implementation of the "integrate health into all policies" strategy. Since the release of the specifications, China CDC has conducted technical specification training for provincial and municipal CDC staff who carry out air pollution and health impact monitoring, standardized air pollution health risk assessment methods, and promoted the extensive development of health risk assessment work. The extensive application of monitoring data in local areas provides a scientific basis for the formulation of air pollution control policies and environmental health management. In addition, it can help all readers better understand the risk assessment results and facilitate improved communication of risks.

Conflicts of interest: No conflicts of interest.

Funding: The National Health and Family Planning Commission of the People's Republic of China (201402022 and 201502003).

doi: $10.46234 / \mathrm{ccdcw} 2021.175$

\# Corresponding author: Dongqun Xu, xudq@chinacdc.cn.

${ }^{1}$ China CDC Key Laboratory of Environment and Population Health, National Institute of Environmental Health, Chinese Center for Disease Control and Prevention, Beijing, China.

Submitted: June 07, 2021; Accepted: August 10, 2021

\section{REFERENCES}

1. GBD 2019 Risk Factors Collaborators. Global burden of 87 risk factors in 204 countries and territories, 1990-2019: a systematic analysis for the Global Burden of Disease Study 2019. Lancet 2020;396 (10258):1223 - 49. http://dx.doi.org/10.1016/S0140-6736(20)307522.

2. Disease Prevention and Control Bureau, National Health Commission, PRC. Work plan for air pollution (Smog) health impact monitoring work program, in 2013. http://www.nhc.gov.cn/jkj/s5898bm/201310/ 02c483b454264c4aa0e99d580fc91c71.shtml. [2013-10-15]. (In Chinese).

3. National Public Service Platform for Standards Information. Industry standard information platform. http://hbba.sacinfo.org.cn/stdList?key= health risk \& ministry=wsjkwyh. [2021-6-9]. (In Chinese).

4. National Health Commission of the People's Republic of China. Technical specifications for health risk assessment of ambient air pollution (WS/T 666-2019). http://www.nhc.gov.cn/fzs/s7848/ 201907/3baff64579a54d5585923a272dfcbcf2.shtml. (In Chinese).

5. World Health Organization. Health risk assessment of air pollution general principles. Copenhagen: WHO Regional Office for Europe; 2016. https://www.euro.who.int/_data/assets/pdf_file/0006/298482/ Health-risk-assessment-air-pollution-General-principles-en.pdf.

6. World Health Organization. WHO Expert Meeting: methods and tools for assessing the health risks of air pollution at local, national and international level. Bonn, Germany: WHO Regional Office for Europe; 2014. https://extranet.who.int/iris/restricted/handle/10665/143712.

7. US Environmental Protection Agency. Guidelines for exposure assessment. Washington, DC: US Environmental Protection Agency, Risk Assessment Forum; 1992. Report No.: EPA/600/Z-92/001. https://cfpub.epa.gov/ncea/risk/recordisplay.cfm?deid=15263. 
8. US Environmental Protection Agency. Guidelines for the health risk assessment of chemical mixtures. Washington, DC: US Environmental Protection Agency, Risk Assessment Forum; 1986. Report No.: EPA/630/R-98/002. https://www.epa.gov/risk/guidelines-health-riskassessment-chemical-mixtures.

9. US Environmental Protection Agency. Guidelines for carcinogen risk assessment. Washington, DC: US Environmental Protection Agency, Risk Assessment Forum; 2005. Report No.: EPA/630/P-03/001F. https://www.epa.gov/risk/guidelines-carcinogen-risk-assessment.

10. US Environmental Protection Agency. A review of the reference dose and reference concentration processes. Washington, DC: US Environmental Protection Agency, Risk Assessment Forum; 2002. Report No.: EPA/630/P-02/002F. https://www.epa.gov/sites/default/ files/2014-12/documents/rfd-final.pdf.

11. US Environmental Protection Agency. Risk assessment guidance for superfund (RAGS), volume I: human health evaluation manual (Part F, supplemental guidance for inhalation risk assessment). Washington, DC: US Environmental Protection Agency; 2009. Report No.: EPA 540-R-070-002. http://www.eugris.info/displayresource.aspx? $\mathrm{r}=6882$. 